\title{
Management of Ebstein's anomaly
}

\author{
Irving L. Kron, Mark Elliot Roeser \\ Division of Thoracic and Cardiovascular Surgery, University of Virginia, Charlottesville, USA \\ Correspondence to: Irving L. Kron, MD. Division of Thoracic and Cardiovascular Surgery, University of Virginia, Charlottesville, USA. \\ Email: ILK@hscmail.mcc.virginia.edu.
}

\begin{abstract}
Ebstein malformation is a rare disease that presents with a spectrum of severity. We use a management paradigm that breaks the anomaly into two groups: neonates and children/adults. This leads to a discussion of management and operative techniques as well as pitfalls. We hope the reader finds our streamlined and logical approach of benefit to this complex and rare disease.
\end{abstract}

Keywords: Ebstein's anomaly; cone repair; Starnes' procedure

Submitted Feb 26, 2017. Accepted for publication Apr 18, 2017.

doi: 10.21037/acs.2017.05.03

View this article at: http://dx.doi.org/10.21037/acs.2017.05.03

\section{Introduction}

We were honored to write this perspective piece in memory of my dear friend, Larry Cohn. He was a true valve repair innovator. The anatomic repair of Ebstein's required as much creativity as any valve procedure. This truly is the essence of Dr. Cohn.

Prenatal diagnosis of the Ebstein malformation has allowed for improvements in immediate postnatal management. Open communication amongst the pediatric intensivist, cardiologist and maternal fetal medicine physician can help risk stratify the patient and decide what monitoring/ level of care will be needed postnatally. If there is any concern for a neonatal intervention, we prefer to have these patients placed in the cardiac pediatric ICU, as they can have severe cyanosis and acidosis due to lung hypoplasia secondary to severe right atrial enlargement in utero and right to left shunting at the foramen ovale. Prenatal awareness and preparation will help to avoid management errors.

The classic chest X-ray in neonatal Ebstein's is a cardiac silhouette that fills the entire chest (a "wall to wall heart") due to right heart enlargement. An echocardiogram should confirm the diagnosis of Ebstein's anomaly, and typically is the only imaging modality needed (CT, MRI and angiography are typically not helpful). Special points of interest during the echo include the size of the right atrium and tricuspid annulus, the fixation of the anterior tricuspid leaflet, the degree of atrialization of the right ventricle (RV) and the remaining cavity of the RV. The anatomy of the RV outflow tract and the pulmonary valve should be scrutinized in addition to the presence of prograde flow out of the right heart to determine if prostaglandin E1 (PGE) infusion is necessary. Even if there is no anatomic obstruction there can be functional pulmonary atresia, as the RV is unable to generate pressure great enough to open the pulmonary valve against the open ductus arteriosus. While rare, significant pulmonary valve insufficiency and tricuspid valve insufficiency with a large atrial shunt and patent ductus arteriosus can lead to a circular shunt.

Knott-Craig has published an algorithmic approach to the treatment of neonatal Ebstein's, and for the most part we follow it. Stable patients are initially treated with supplemental oxygen, observation for adequate cardiac output, and possible PGE infusion to ensure pulmonary blood flow. In an unstable patient, intubation and sedation, along with inotropic support and PGE can help improve cardiac output, decrease metabolic demand and ensure pulmonary blood flow. Other therapies to decrease pulmonary vascular resistance and promote blood flow through the RV, such as oxygen, nitric oxide and avoiding acidosis can be helpful. We believe that paralysis with volume-cycled ventilation $(12-15 \mathrm{~mL} / \mathrm{kg}$ per breath) is helpful in minimizing the effect of gross cardiomegaly on cardiac output (1). If the pulmonary valve is patent and the patient can be stabilized, then the best course of action is to wait until the pulmonary vascular resistance drops and more 
blood flow can flow through the RV into the pulmonary arteries. It may take multiple attempts and several weeks, but many patients can be treated conservatively, weaned off PGE and have their repair pushed out of the neonatal period.

If anatomic or functional pulmonary valve atresia exists or the patient is dependent on PGE infusion, surgery is required. Patients with a small RV and mild tricuspid regurgitation (TR) should have a modified Blalock-Taussig shunt (mBTS). The mild TR and right-to-left shunting at the atrial level is a marker for inadequate RV size (1) and these children should follow the single ventricle route with a heightened awareness of aberrant conduction pathways causing supraventricular tachycardia. If there is moderate to severe TR and the functional RV is small, then a fenestrated right ventricular exclusion (Starnes' Procedure) should be performed. The goals of this procedure are to exclude and decompress the malformed $\mathrm{RV}$, perform a reduction atrioplasty, ensure a wide open atrial septal defect (ASD), and provide pulmonary blood flow via an mBTS (2).

\section{Starnes' procedure}

This procedure has evolved to include a fenestration in the patch that excludes the functional $\mathrm{RV}$, ensuring drainage of the coronary sinus into the right atrium, and relief of any pulmonary artery (PA) regurgitation. After sternotomy and thymectomy, the enlarged right atrium is always obscuring the dissection. A 3-0 silk retraction tie on the right atrial appendage can help with dissection of the aortopulmonary (AP) window and the superior vena cava (SVC). However, if the enlarged right atrium is still a nuisance, a single venous cannula should be placed and cardiopulmonary bypass (CPB) initiated before dissecting out the SVC and inferior vena cava (IVC). The ductus is ligated shortly after going on $\mathrm{CPB}$ and the patient is converted to bicaval cannulation. After cross clamping and arrest, the incision for the atrial reduction is made on the anterior surface of the right atrium, making sure to allow for a simple linear closure with no tension on the cavae and an adequate margin on the atrioventricular (AV) groove (2).

The internal anatomy of the right atrium should be carefully scrutinized. Ventral traction on the atrial free edge should identify the "anatomic" tricuspid annulus. The coronary sinus should also be clearly identified with a dose of cardioplegia. The atrial secundum membrane is excised and the favored patch material is trimmed to the "anatomic" tricuspid annulus. The patch is sewn into place using prolene sutures, making sure the right coronary is not injured or torsed in the AV groove. Special care is taken around the conduction system and sutures should avoid the mouth of the coronary sinus. Pathologic studies have shown that the AV node may be displaced toward the ostium in Ebsteinoid hearts (3) - frankly, there is no reason to be in that area. If the septal leaflet will hold stitches, we prefer to sew the patch to it and avoid the conduction system altogether. The fenestration is performed with a $4 \mathrm{~mm}$ punch. This decompresses the RV from any drainage from the Thebesian veins and relieves the septal impingement on the left ventricle (LV) seen in these massively dilated hearts (2). If there is pulmonary insufficiency, then the PA must be interrupted. An mBTS is then performed.

If there is moderate to severe TR and an adequate sized functional $\mathrm{RV}$, then a complete biventricular repair may be considered. In this instance, the heart team has decided that the $\mathrm{RV}$ is of adequate size and that the tricuspid valve can be repaired in the setting of neonatal pulmonary vascular resistance. In order to accomplish this, a RV to PA (RV-PA) valve conduit should be used. Knott-Craig reports a $46 \%$ mortality with a transannular patch. Limiting the incision on the RV for a small (about 7-10 mm) conduit will help the struggling $\mathrm{RV}$, as will paralysis and nitric oxide. This is not only a delicate surgical repair, but it will leave a tenuous patient in the ICU for a prolonged postoperative course with a less than ideal $R V$, a newly repaired tricuspid valve, and a ventriculotomy. Single ventricular palliation may be better than a struggling biventricular repaired neonate.

\section{Neonatal biventricular repair}

The goals of this operation are the creation of a competent tricuspid valve, right atrioplasty, closure of the ASD with creation of a small fenestration, repair of all associated defects, and placement of a valved RV-PA conduit (1). After bicaval $\mathrm{CPB}$ is achieved and cardioplegia given to arrest the heart, a right atrioplasty is performed as described above. If the RV appears adequate on inspection, we prefer to plan the positioning of RV-PA conduit at this time. A right angle is placed through the tricuspid valve and a position on the infundibulum is marked on where the incision is to be made. We then cut any muscle bundles that may impair flow to the conduit. Next, the tricuspid valve is heavily scrutinized (particularly the leading edge of the anterior leaflet). The repair is based on a monocusp valve using the anterior leaflet. If the leaflet is "sail like" and free, but the annulus is severely dilated, then an aggressive Kay annuloplasty is performed. A hegar dilator of the correct 
$\mathrm{z}$-score is placed in the tricuspid orifice and pledgetted suture is placed through the anterior-posterior commissure and brought out just below the coronary sinus making sure the left side of the orifice is not smaller than the dilator (4). This creates a double orifice tricuspid valve. The right orifice is then closed with sutures, leaving the left orifice with the anterior leaflet acting as a monocusp valve. The repair is saline tested and additional sutures between the anterior and septal leaflets may need to be placed to shore up any leaks. Once the repair is adequate, we will proceed with the RV-PA conduit. We prefer to use a pericardial gusset at the proximal anastomosis.

In patients with functional pulmonary atresia and a small $\mathrm{LV}$ size, transplant is the only viable option. In patients with adequate $\mathrm{LV}$ size who cannot be stabilized, a Starnes palliation or mBTS is performed (1). The main PA may need to be ligated if there is concern for a circular shunt. In those patients that are stable, medical therapy is trialed as described previously. If the neonate is unable to be weaned from prostaglandins and inhaled nitric oxide, then surgery is necessary. Those with mild TR and an inadequate RV will need an mBTS and will be directed down the single ventricle pathway. Those with moderate to severe TR and an adequate functional RV size can undergo a complete biventricular repair.

\section{Child/adult}

Children who have survived infancy generally do well for several years and surgery can be postponed until symptomatic: exercise capacity begins to deteriorate, paradoxical embolism occurs, cyanosis (oxygen saturations $<90 \%$ ) occurs, progressive right ventricular enlargement by echocardiography or onset or progression of atrial or ventricular arrhythmias. The continual refinement of echocardiography has made it easier to assess the probability of a successful repair preoperatively, and this also factors into surgical timing. We will sometimes perform a bidirectional Glenn procedure to "volume unload" the RV and improve the likelihood of satisfactory repair.

After the median sternotomy, the RV and right atrium are inspected to detect any dilation of the atrialized RV. The right atrial appendage is usually bulging and a tie helps to retract it out of the way. Care should be taken to place the cannula high in the SVC in case a Glenn shunt needs to be performed. After CPB is initiated, the thickness of the $\mathrm{RV}$ is evaluated. Chauvaud suggest that a paper thin right ventricular wall is an indication that a Glenn shunt might be needed (5). Like most surgeons, we use mild hypothermia
(32-34 degrees Celsius) and prefer to perform the repair with a complete cardiac arrest. Evaluation of the anomaly includes examination of the dilated annulus, evaluation of the delamination of the valve leaflets, and atrialization of the RV. The infundibulum is then exposed with a retractor and the leaflets pulled centrally to expose the abnormal bands connecting them to the RV.

\section{Cone reconstruction}

The cone reconstruction is our preferred technique. It is different from previous valvuloplasty techniques in that it is closest to an "anatomic repair" (6). The caveat is that there must be greater than $50 \%$ delamination of the anterior leaflet and a usable posterior leaflet. The end result is 360 degrees of leaflet tissue. After the tricuspid leaflets are inspected, stay sutures are placed on the annulus of the anterior, posterior and septal leaflets, being careful not to injure the conduction system. The anterior leaflet is incised from its annulus to expose the attachments to the leading edge. All attachments to the body of the leaflet are excised, but those attached to the leading edge are kept. Any linear attachments to the leading edge are fenestrated, in order to obtain mobility of the anterior leaflet. Instead of stopping at the anteroposterior commissure, this incision is continued to the posterior leaflet. Great care and time is taken to then free the posterior leaflet from the underlying myocardium. All attachments to the body of the leaflets may be cut, but the attachments to the free edge must be left intact. This is similar to what is performed on the anterior leaflet except that the delamination of the posterior and septal leaflets is usually less than that of the anterior leaflet, making this dissection cumbersome. When the leading edge is attached to the myocardium, fenestrations are performed to help free the leaflet. The height of the fenestrations is typically onefourth to one-third the distance from the leaflet edge (6). Care must be taken avoid injury to the thinned underlying $R V$. The septal leaflet can then be separated from its "annulus" and the attachments to the body of the leaflet freed. Careful attention is paid to the area of the conduction system during this process. The septal leaflet is usually very fragile and thin, especially as one heads to the anteroseptal commissure. The septal leaflet is always the most underdeveloped and the most frustrating. At this point, all three leaflets have been mobilized with only the free edge attached to the myocardium. The septal leaflet will sometimes be diminished at the anteroseptal commissure and a commissuroplasty will help make up for inadequate 
leaflet tissue. This can also be performed with the posterior and septal leaflets. It also ensures 360 degrees of leaflet tissue that will make up the new tricuspid valve (6). This creates support for both the anterior and newly constructed septal leaflet as well as transforming the shallow but wide tricuspid valve into a narrower but taller valve. Or taking a wide and shallow cone and creating a taller but narrower cone using the same amount of material.

With the newly constructed leaflets complete, the atrialized $\mathrm{RV}$ is examined. The section of $\mathrm{RV}$ that usually needs to be remodeled includes the acute margin near the diaphragmatic surface. Identification of the right coronary artery and posterior descending artery is critical to avoid injury or kinking during the plication. The goal of the plication is to reduce the volume of the ventricle as well as removing akinetic and dyskinetic regions to improve efficiency. It also reduces the distraction of the papillary muscles and primary chordal attachments, which contribute to recurrent TR (7). The plication is taken up to the level of the true tricuspid annulus. Since the newly constructed valve has a smaller diameter than the annulus, it is plicated on the inferior aspect with figure of eight or interrupted sutures. The leaflets are then reattached to the true annulus with a running fine prolene suture. We reinforce the repair with an annuloplasty ring and believe this is important [as shown by the Mayo group (5)].

Unless the repair was easy, we leave a $4 \mathrm{~mm}$ fenestration in the ASD. A thin walled RV with poor function is a preoperative indication that a superior cavopulmonary shunt may be needed. If there is significant tricuspid stenosis or failure to wean from bypass with an LA pressure less than 10 and CVP greater than 15, with low systemic pressures, than a superior cavopulmonary shunt is performed (8). We prefer to do selective right sided cryo-ablation-Maze for patients with documented paroxysmal flutter or inducible atrial arrhythmias. Some surgeons, however, will perform this on all patients undergoing operative repair.

I think it is important to note that a good replacement is better than a poor tricuspid valve repair. Between 1972 and 2006, Mayo performed 519 initial operations for Ebstein's anomaly, with 182 repairs and 337 replacements (9). Upon reviewing these patients, having a valve replacement did not impact their survival or functional outcome, and it did not statistically raise their risk of reoperation. Having a reduction atrialplasty was associated with an excellent or good functional outcome and a preoperative history of arrhythmia was associated with a poorer outcome. In fact, arrhythmias were the most common cause of rehospitalization.

\section{Acknowledgements}

None.

\section{Footnote}

Conflicts of Interest: The authors have no conflicts of interest to declare.

\section{References}

1. Knott-Craig CJ, Goldberg SP, Ballweg JA, et al. Surgical Decision Making in Neonatal Ebstein's Anomaly: An Algorithmic Approach Based on 48 Consecutive Neonates. World J Pediatr Congenit Heart Surg 2012;3:16-20.

2. Reemtsen BL, Starnes VA. Fenestrated right ventricular exclusion (Starnes' procedure) for severe neonatal Ebstein's anomaly. Oper Tech Thorac Cardiovasc Surg 2008;13:91-100.

3. Celermajer DS, Cullen S, Sullivan ID, et al. Outcome in neonates with Ebstein's anomaly. J Am Coll Cardiol 1992;19:1041-6.

4. Knott-Craig CJ. Management of neonatal Ebstein's anomaly. Oper Tech Thorac Cardiovasc Surg 2008;13:101-8.

5. Chauvaud S. Repair of Ebstein's anomaly. Oper Tech Thorac Cardiovasc Surg 2004;9:194-207.

6. Dearani JA, Bacha E, da Silva JP. Cone reconstruction of the tricuspid valve for Ebstein's anomaly: anatomic repair. Oper Tech Thorac Cardiovasc Surg 2008;13:109-12.

7. Emani SM, Del Nido P. Chapter 101: Ebstein's Malformation of the tricuspid valve in children. In: Kaiser LR, Kron IL, Spray TL. Editors. Mastery of Cardiothoracic Surgery, 3rd edition. Baltimore: Lippincott Williams \& Wilkins, 2015:1098-104.

8. Anderson HN, Dearani JA, Said SM, et al. Cone reconstruction in children with Ebstein anomaly: the Mayo Clinic experience. Congenit Heart Dis 2014;9:266-71.

9. Brown ML, Dearani JA, Danielson GK, et al. Functional status after operation for Ebstein anomaly: the Mayo Clinic experience. J Am Coll Cardiol 2008;52:460-6.

Cite this article as: Kron IL, Roeser ME. Management of Ebstein's anomaly. Ann Cardiothorac Surg 2017;6(3):266-269. doi: 10.21037/acs.2017.05.03 